\title{
ENSO Bred Vectors in Coupled Ocean-Atmosphere General Circulation Models
}

\author{
S.-C. YAng, $*$ M. Cai, ${ }^{+}$E. Kalnay, $*$ M. Rienecker, ${ }^{\#}$ G. Yuan, ${ }^{@}$ And Z. Toth ${ }^{\&}$ \\ *Department of Meteorology, University of Maryland, College Park, College Park, Maryland \\ + Department of Meteorology, The Florida State University, Tallahassee, Florida \\ \# Global Modeling and Assimilation Office, NASA GSFC, Greenbelt, Maryland \\ @ Bauer Center for Genomics Research, Harvard University, Cambridge, Massachusetts \\ \& Environmental Modeling Center, NOAA/National Centers for Environmental Prediction, Camp Springs, Maryland
}

(Manuscript received 12 October 2004, in final form 13 September 2005)

\begin{abstract}
The breeding method has been implemented in the NASA Seasonal-to-Interannual Prediction Project (NSIPP) coupled general circulation model (CGCM) with the ultimate goal of improving operational seasonal to interannual climate predictions through ensemble forecasting and data assimilation. This is the first attempt to isolate the evolving ENSO instability and its corresponding global atmospheric response in a fully coupled ocean-atmosphere GCM. The results herein show that the growth rate of the coupled bred vectors (BVs) is sensitive to the ENSO phases of the evolving background flow and peaks about 3 months before an ENSO event. The structure of the dominant growing BV modes also evolves with the background ENSO and exhibits a larger amplitude in the eastern tropical Pacific, reflecting the natural dynamical sensitivity associated with the shallow thermocline at the eastern boundary. The key features of coupled bred vectors of the NSIPP CGCM are reproduced when using the NCEP CGCM, an independently developed coupled general circulation model.
\end{abstract}

\section{Introduction}

The El Niño-Southern Oscillation (ENSO) phenomon is responsible for a large portion of interannual iability in the tropical Pacific. ENSO also has a globimpact in climate anomalies and extreme weather ents. Feedbacks through atmosphere-ocean coupling the Tropics characterize the covariability of wind, $T$, and thermocline (or warm water volume) of ISO. Many features of the ENSO events have been blained by the delayed oscillator mechanism owing to pagating downwelling/upwelling information in the per ocean associated with equatorial waves (Schopf d Suarez 1988; Suarez and Schopf 1988; Battisti 38). Jin (1997) further emphasized the importance of variations of warm water volume in the upper ocean h a warm water recharge/discharge mechanism. It been shown that the coupled dynamic/thermodynic mechanisms, that is, the thermocline and Ekman dbacks, responsible for delayed or recharge/discharge

Corresponding author address: Dr. Ming Cai, Department of teorology, The Florida State University, Tallahassee, FL 06.

oscillators, can explain both the west-east asymmetry in the climate mean state and the ENSO variability in the equatorial Pacific basin (Cai 1995; Jin 1996; Dijkstra and Neelin 1999; Van der Vaart et al. 2000; Cai 2003). The dynamical atmosphere-ocean coupled models constructed with the concept of the delayed or recharge/ discharge oscillator have shown valuable prediction skill for the ENSO events (Cane et al. 1986; Zebiak and Cane 1987). The strong weather and climate impact from ENSO has motivated the advancement of prediction tools from simple anomaly coupled models to fully coupled global ocean-atmosphere general circulation models (CGCMs).

Factors that influence the skill in forecasting seasonal-interannual SST anomalies include the errors in oceanic and atmospheric initial conditions, atmospheric stochastic variability, and model errors. It is known that the presence of errors in the initial condition limits the ENSO prediction skill (Latif et al. 1998) and that an ensemble forecast should sample the uncertainties in the initial condition (Stockdale et al. 1998). Vialard et al. (2003) performed a series of ensemble forecast experiments with a state-of-the-art coupled GCM using wind and SST perturbations and stochastic physics, in- 
hat the uncertainties in SST determine the spread emble forecasts in early months of the forecast, perturbations of the wind stress or atmospheric al variability are less efficient in generating SST ions. These results are supported by Chen et al. who argue that the evolution of El Niño is con$d$ to a large degree by self-sustaining internal dy$\mathrm{s}$ in the Tropics. These results suggest that the prediction depends more on initial conditions on unpredictable atmospheric noise and that there od reason to investigate the inclusion of coupled perturbations for ensemble ENSO prediction. he ENSO ensemble prediction, the challenge is o generate ensemble perturbations that can effecrepresent SST uncertainties in seasonal-interancale in a CGCM. Moreover, initial ensemble perions need to be constructed in a coupled manner ler to ensure that they reflect the uncertainties ated with the coupled instability. In addition, ene perturbations for ENSO prediction should also t the uncertainties in teleconnected atmospheric les to improve coupled forecast skill beyond the al Pacific region.

thods for generating ensemble perturbations can osely categorized into linear and nonlinear aphes. The singular vector linear approach looks for timal initial perturbations that will maximize the $\mathrm{h}$ of the perturbations after a chosen period with sen norm. With an optimization time of 3-6 Is and the choice of the SST norm, Penland and shmukh (1995), Chen et al. (1997), and Thomp998) showed that a final singular vector with large tude of the SST signal is located in the southeastacific. It should be pointed out that, even though rowth rate of their singular vector strongly deon seasonal cycle and ENSO phase, their initial ar vectors are insensitive to both. However, also a SST norm, Xue et al. (1994, 1997a,b) and Fan et 000) obtained ENSO-like initial singular vectors, asizing that the signals in the eastern Pacific are concentrated toward the equator, even though final singular vectors are very similar to those in Chen et al. (1997) and Thompson (1998). Fia very different pattern compared to all of the studies has been obtained from a series of studies by Moore and Kleeman (1996, 1997, 1999a,b, Their results emphasize large signals in the west atral Pacific. When considering a multivariable bation norm, Fan et al. (2000) and Moore and han (2001) reached different conclusions on the ve importance between the SST and the therne. The strong dependence on the choice of norm conclusion as to their use for ENSO ensemble perturbations. Moreover, with a very complex system like a CGCM that includes a wide range of instabilities, leading singular vectors would be dominated by the fast growing error related to weather and even convection (Peña and Kalnay 2004). Thus, to obtain an ENSOrelated singular vector in a coupled GCM, one needs to exclude less relevant fast growing components explicitly from the tangent linear operator (Kleeman et al. 2003). The computational/development costs are also a limitation for the singular vector method since it requires constructing coupled tangent linear and adjoint operators.

The breeding method proposed by Toth and Kalnay $(1993,1997)$ is designed to estimate the shape of the growing dynamic error using the full nonlinear model. This method takes advantage of the early nonlinear saturation of convective instabilities compared to baroclinic instabilities. Toth and Kalnay (1996) suggested this advantage could be applied in a coupled oceanatmosphere system to isolate ENSO coupled instabilities from the faster weather-related instabilities. Cai et al. (2003) first tested the breeding method in a coupled system using the ZC model (Zebiak and Cane 1987). They found that the bred vector (BV) growth rate is weakest at the peak time of the ENSO states (both positive and negative) and strongest between the events. Also, the coupled bred vectors are insensitive to the choice of norm but very sensitive to the background ENSO phase and the annual cycle. It should be pointed out that the ZC model only has ENSO-related instability and the fast processes are already explicitly excluded from the ZC model. Peña and Kalnay (2004) illustrated the idea that breeding is able to isolate the slow modes of a coupled system when rescaling intervals and amplitudes are chosen from physically appropriate scales and the rescaling factor is obtained from the slow component of the system. Independent work by Boffetta et al. (1998) also demonstrated that choosing the perturbation size is a powerful tool to isolate the slow mode in a system with multiple time scales.

The results with the simple $\mathrm{ZC}$ model encouraged us to implement the breeding method in a CGCM, without sacrificing resolution or simplifying model physics. The new challenge is that a CGCM includes many types of instabilities with different time scales. Therefore, we need to demonstrate, as a first step, whether we can obtain the slowly growing coupled instabilities as in Cai et al. (2003) using breeding in a comprehensive CGCM. Our objective is to identify the characteristics of bred vectors associated with the ENSO derived from the CGCM and to investigate whether an ENSO-related 
tabilities using the breeding method. Specifically, in s paper, we address the following questions. 1) Can eding be used to identify or detect the coupled, wly growing ENSO instability and isolate it from er short-term instabilities? 2) Is the coupled BV de sensitive to the ENSO phases? and 3) Are the in characteristics of coupled bred vectors reproducwith different coupled ocean-atmosphere GCMs? The paper is organized as follows. In section 2, we e a brief description of the National Aeronautics and ace Administration's (NASA's) Seasonal-toerannual Prediction Project (NSIPP) coupled model it has been used to generate coupled bred vectors. e simulated ENSO variability in the NSIPP model is o included in section 2. Section 3 describes how the eding method is applied in a coupled GCM for isoing the slowly varying coupled instability. Section 4 cribes the main characteristics of coupled bred vec$\mathrm{s}$ derived in the NSIPP model. A comparison of the ults obtained from NSIPP and from the National nters for Environmental Prediction Coupled Foret System Model (NCEP/CFS03) is also presented in tion 4. A brief summary and discussion of the next ase of our research are included in section 5 .

\section{Breeding method with the NSIPP coupled global circulation model}

n this study, we implemented the breeding method the NSIPP coupled ocean-atmosphere general ciration model. The NSIPP coupled model is a fully upled global ocean-atmosphere-land system develed at the NASA Goddard Space Flight Center SFC) (Vintzileos et al. 2003). It comprises the NSIPP nospheric model (AGCM) (Bacmeister and Suarez 2); Bacmeister et al. 2000), the Poseidon ocean del (OGCM) (Schopf and Loughe 1995; Yang et al. 9), and the Mosaic land surface model (LSM) (Ko$r$ and Suarez 1992). The official NSIPP Web site tains detailed information about the NSIPP coupled del (http://nsipp.gsfc.nasa.gov/).

A 62-yr simulation run had been made with a rerch version of the NSIPP CGCM with a resolution $3^{\circ}$ latitude by $3.75^{\circ}$ longitude and 34 sigma layers in AGCM and $1 / 2^{\circ}$ latitude by $1.25^{\circ}$ longitude and 27 ers in the OGCM. This integration constitutes our ature run" and is referred to as the "background" in s paper. The bred perturbations are grown upon the lving background flow. The wind stress, SST, and rmocline anomalies from this long simulation exit coupled ENSO interannual variability. However, ENSO cycle of the control run has an unrealistically ong biennial component rather than the observed amplitude

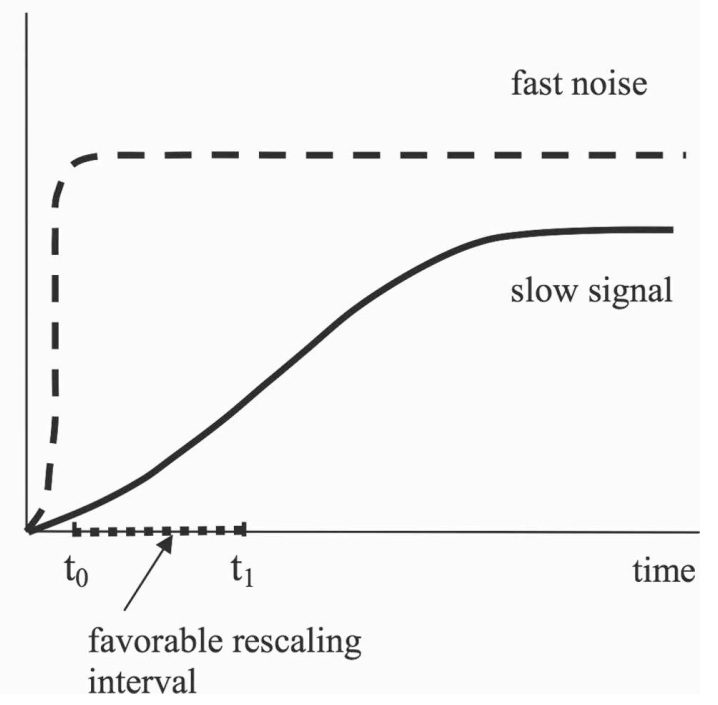

FIG. 1. Schematic showing that, if the rescaling time interval for breeding is larger than $t_{0}$, the saturation time of the fast noise, and smaller than the time $t_{1}$, when nonlinearities reduce the growth of the slow signal, the relative amplitude of the linearly growing slow signal is boosted with respect to the noise.

viation of the SST anomaly in the eastern Pacific is only half of the observed. This problem has been attributed to the atmospheric longitudinal circulation near the equator (Rienecker et al. 2000). The spatial patterns of the coupled ENSO variability of the background are shown in the left panels of Figs. 4-6 and will be discussed together with the bred vector spatial structure in section 3 .

We used a 10-yr model simulation run to perform breeding experiments under a "perfect model scenario," that is, assuming that the unperturbed simulation run is the "truth" (or analysis in a forecast system). The breeding method (Toth and Kalnay 1993, 1996, 1997) was originally developed to "breed" growing modes related to synoptic baroclinic instability for short-to-medium range atmospheric ensemble forecasting by taking advantage of the fact that small amplitude, but faster growing, convective instabilities saturate earlier. The idea that different amplitudes and time scales characterize different types of instabilities is applied to isolate the slowly growing coupled ENSO instability from the fast growing weather signal (see schematic Fig. 1). This is difficult to do using atmospheric variables since the amplitude of the weather noise is larger than that of the ENSO-related coupled signal. The slow, coupled perturbation can only be isolated by measuring the growing perturbations using the slowly evolving component of the coupled system, namely the 
the period longer than two weeks is important in to estimate the slowly varying coupled instability turate the growth of the weather signal (Peña and y 2004).

procedure of breeding cycles in a coupled system en described in Cai et al. (2003). Bred vectors are eriodically rescaled difference between the per$d$ run and the nonperturbed run (background). escaling period (longer than two weeks) is chosen w fast weather instabilities to saturate well before scaling time, whereas the slowly evolving ENSO ility is still growing (Fig. 1). Both oceanic and pheric perturbations are scaled down with the factor, measured by the growth of the ocean comt. This gives a relative advantage to the slowly ng ENSO mode (as well as to any other slow inty present in the coupled system). In the next ing cycle, the saturated fast instability perturbaarts with an unfavorable shape and does not have room to grow, but the slow ENSO instability from a favorable (linear) shape that can still grow volve. Therefore, by repeating the breeding proe (periodically resizing the perturbation and addto the background) we can enhance the relative tude of the slow instability and allow the bred bation to align along favorable growing direcAlthough this does not completely isolate the perturbation, it gives it a relative boost comto faster growing perturbations that saturate 1), but other instabilities with time scales longer he weather instabilities will be also present in the $n$ the next section, we focus on the dynamic struc$f$ the coupled bred vector associated with the evoof the background, which is viewed as "nature" erfect model scenario, since in this study we are oncerned with model error.

breeding method was implemented in the NSIPP $M$ with a 1-month rescaling period and using the ifference in the Niño-3 SST within the tropical c domain $\left(15^{\circ} \mathrm{S}-15^{\circ} \mathrm{N}, 120^{\circ} \mathrm{E}-90^{\circ} \mathrm{W}\right)$ as the rescalrm. The rescaling amplitude of the rms SST was n to be $0.085^{\circ} \mathrm{C}$, or about $10 \%$ of the background ariability. The 1-month rescaling period, which is longer than the time scale of the atmospheric linic instability, is also a convenient choice bein the NSIPP system users can access only nly model output. According to the experience in al. (2003) with the ZC model, the structure of the vector is rather insensitive to either the choice of scaling period (if it is long enough to saturate the er signal) or the choice of the slow variable norm $r$ the oceanic energy norm). Also, breeding ex- with the NCEP coupled GCM using 15 days as the rescaling period have a similar behavior of the $\mathrm{BV}$ growth rate as with a 1-month rescaling period. The rescaling norm must be associated with the ocean component in order to capture the slowly growing instability. Since the SST directly represents the strength of the ENSO variability, it is natural to use it to detect the growing coupled instability. Because the research version of the NSIPP coupled GCM used in this paper is no longer available, it was not possible to test different breeding rescaling norms. However, tests with the operational version of the NSIPP coupled GCM show that the BV structure obtained is robust when using either a SST norm measured in the Niño-3 region or a thermocline norm measured in the tropical Pacific.

Two independent breeding runs were made starting from two perturbations created by taking the difference between two model states at randomly chosen months. Each run contains 123 months, starting from September of the 19th year to December of the 29th year of the $62-y r$ simulation run. The starting month is chosen to be well separated from the first major warming event, which takes place about 2 years into the breeding run. This is to ensure that the breeding experiment does not start when the background is dominated by the mature ENSO condition. Two independently generated breeding experiments show that bred vectors start to have similar structure after three months. Thus, we treat the first three months as a transient period allowing the bred vectors to align along the dynamics-dominated instability. The analysis presented below is derived from the remaining 120 breeding cycles (120 months). We found that two independent 10-yr breeding cycles yielded very similar bred vectors (not shown), so we combine the two bred vector perturbations as a single time series of 20 years to reduce sampling errors. Hereafter, we refer to the combined bred vectors as BV perturbations.

\section{Results}

\section{a. Growth rate of coupled bred vectors}

Bred vectors represent, by construction, the instabilities that have been growing in the recent past upon the background flow. In addition to ENSO, our method captures any instability with a time scale longer than 2 weeks. An example of such instability is shown in Fig. 2, a snapshot of the bred vector SST field (contours) together with the corresponding background SST field (shading) on 1 July of model year 24. It shows that the bred vector field has a large amplitude along the sharp zonal temperature gradient in the equatorial cold tongue, where tropical instability waves are present in 


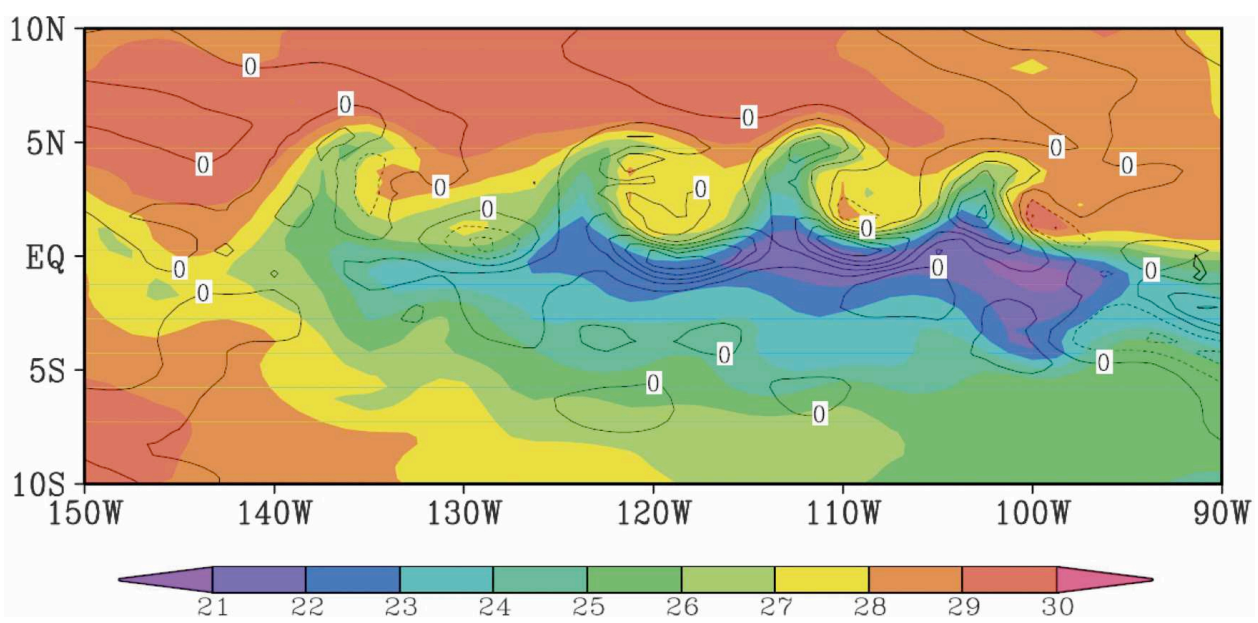

FIG. 2. A snapshot of SST in the eastern Pacific showing the bred vectors' perturbation (contours: CI $=0.15^{\circ} \mathrm{C}$ ) evolving with the background flow (shading with an interval of $1^{\circ} \mathrm{C}$ from $21^{\circ}$ to $30^{\circ} \mathrm{C}$ ) on 1 July of model year 24 . The dotted contours of the BV indicate negative values.

d perturbation is aligned with the background flow such a way as to increase the amplitude of the waves. opical wave instability is active when the cold tongue vell established, like the fall season and the La Niña ents, in both the background and the BV fields. The $t$ that the bred vector can capture the formation of pical instability waves suggests BV sensitivity to the blution of the background flow since the formation of h instability is strongly influenced by seasonal and erannual variability (Contreras 2002). We found that BV structures related to the tropical instability ves disappear when the background is evolving tord a warm anomalous state. This example indicates t we can view the growth of the perturbations as a ult of the superposition and competition among inbilities with different physical mechanisms and time les. To analyze the ENSO component among all wth signals, we examine the relationship between $\mathrm{BV}$ growth rate and the background ENSO varility, described by the Niño-3 index.

The growth rate of the coupled bred vectors is calated based on the chosen rescaling norm of the perbation field within the tropical Pacific region:

$$
G(t)=\frac{\sqrt{\sum_{\mathrm{NG}}\left[\mathrm{BV}_{\mathrm{SST}}(t)\right]^{2}}}{\sqrt{\sum_{\mathrm{NG}}\left[\mathrm{BV}_{\mathrm{SST}}(t-1)\right]^{2}}},
$$

ere NG is the total number of model grid points in Niño-3 region and $t$ is the model time in months. In ter words, we measure the growth rate of bred vec$\mathrm{s}$ by their amplification factor in the Niño-3 region hin a month. In the $10-\mathrm{yr}$ experiment, the mean of deviation is 0.7 . The mean growth rate is much higher than the result obtained from Cai et al. (2003), which has an ENSO growth rate ranging between 1 and 3 . This is to be expected since the presence of additional instabilities in the coupled GCM contributes to the total growth of the perturbations, compared to the $\mathrm{ZC}$ model. For example, the tropical wave instability shown in Fig. 2 grows vigorously but with a shorter time scale than the ENSO variability.

To test whether there is a component of the perturbation growth (above the background noisy growth rate of about 3 per month) evolving upon the coupled ENSO background state (rather than growing randomly), we calculate the lag/lead correlation between the growth rate and the absolute value of the background Niño-3 index. We use the absolute value of the Niño-3 index in order to account for the large amplitude of both positive and negative SST anomalies. It is evident in Fig. 3 that the growth rate of coupled bred vectors tends to be largest about 3-4 months prior to the time when the background ENSO amplitude reaches its maximum stage (positive or negative).

Since the coupled GCM contains different types of instabilities, the correlation level of 0.22 in Fig. 3, although small, is significant in the context of the relative amplitude of the ENSO variability. To test statistical significance, we constructed the correlation between 1000 randomly generated time series and the absolute value of the background Niño-3 index. Each time series has the same mean and variance as the time series of the bred vector growth rates. Among the 1000 random samples, the mean correlation value is 0.017 and the standard deviation is 0.07 . The accumulated percentage 


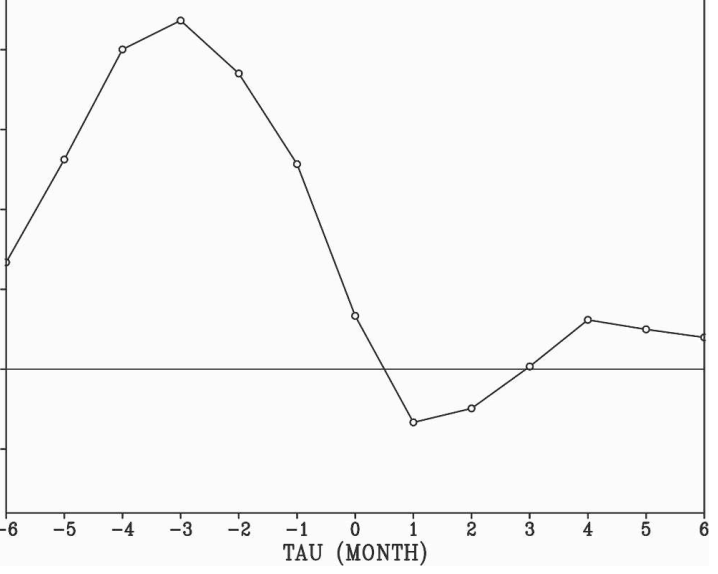

Lead/lag correlations between the BV growth rate and the absolute value of the background Niño-3 index.

level is close to $99 \%$ in stating that a 0.2 correvalue represents a significant correlation to the round Niño-3 index. Therefore, the maximum in supports the conclusion that the bred vector $\mathrm{h}$ rate is related to and leads the background 3 index.

addition, the main growth signal takes place behe ENSO events in Fig. 3 because, by taking the ite value of the background ENSO index in coms the correlation we consider the ENSO phases and treat the cold and the warm cases as if they the same. In Cai et al. (2003), the available time was much longer so that the background Niño-3 for the ZC model was composited over many and the results arranged evolving from the cold to the warm stage. This resulted in the peak of the vector growth rate split equally before and after NSO event. We can conclude that our results are atively in good agreement with those obtained the ZC model in Cai et al. (2003). The results here suggest that the breeding method can serve ect the slow, coupled instability related to ENSO ility by selecting the proper rescaling parameter breeding cycle. Evans et al. (2004) also showed ne bred vector growth rate could be used to detect rthcoming regime changes of the background state positive to negative conditions or vice versa).

\section{e structure of the coupled $B V$ mode}

spatial patterns of the ENSO coupled BV ig all the instabilities present in the system) can entified by constructing regression maps for both ic and atmospheric variables against the $\mathrm{BV}$
SST in the Niño-3 domain. Such spatial structure of the bred vector indicates the linear response related to the ENSO instability and makes the low-frequency variations associated with ENSO clearer. We will compare the ENSO BV maps with the background ENSO regression maps constructed with the same regression method but using the background Niño-3 index. This will allow us to assess whether the coupled $\mathrm{BV}$ has a dominant ENSO growing component, whether it projects on the background ENSO variability, and what physical mechanisms are suggested by the BV ENSO patterns.

The oceanic global regression maps for the background fields show typical tropical variability corresponding to the ENSO mature stage (Figs. 4a-c). These patterns include a large warming extending from the east to central equatorial Pacific, a deepening thermocline in the eastern equatorial Pacific, an accompanying shoaling feature off the equator in the western basin, and a basinwide eastward current anomaly. The regression maps for the $\mathrm{BV}$ fields (regressed upon the BV Niño-3 index) are shown in Figs. 4d-f. The coupled $\mathrm{BV}$ mode exhibits a strong signal in the equatorial $\mathrm{Pa}-$ cific and fairly weak variability away from the Tropics. The patterns of the coupled BV mode are reminiscent of those in the background state except that the BV mode is more confined to the east and to the equator. This feature is physically meaningful since it reflects that the dynamical growing perturbation is mainly determined by the background structure of the thermocline. It is also consistent with the delayed oscillator theory, which considers that the perturbations grow primarily over the eastern equatorial basin through the proportionality between wind stress and the displacement of the thermocline (Cane et al. 1990). The shoaling thermocline in the east implies that the thermodynamic feedback between SST and near-surface ocean variables is much stronger in the east than in the west. As a result, oceanic perturbations in the eastern basin will be easily amplified through positive feedbacks from air-sea interaction. Although we have obtained the ENSO BV by regression with the BV Niño-3 index, Figs. $4 \mathrm{~d}-\mathrm{f}$ can also be referred to as the leading BV mode because they have the same structure as the leading mode obtained by applying EOF analysis to the oceanic BV (as shown in Figs. 8a,b).

The atmospheric components of the ENSO mode derived from both the background and the BV field are displayed in Fig. 5. In the same way that the background regression maps shown in Figs. 5a-d reflect the typical atmospheric response of ENSO events, the BV atmospheric regression map also exhibits the coupled feature corresponding to the boundary heating pertur- 
(a) Regressed CNT SST anomaly

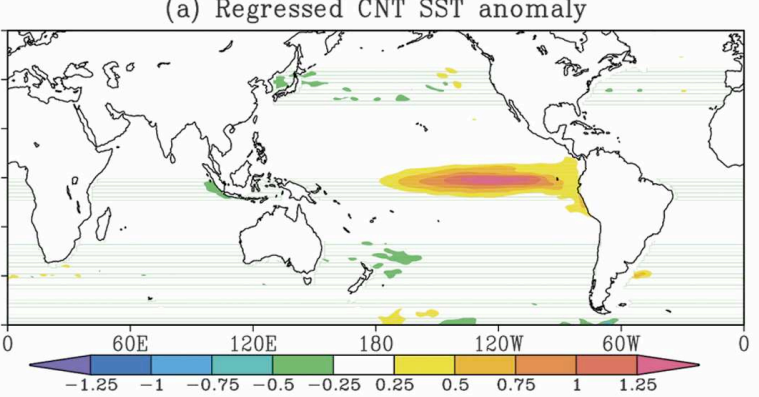

(b) Regressed CNT Z20 anomaly

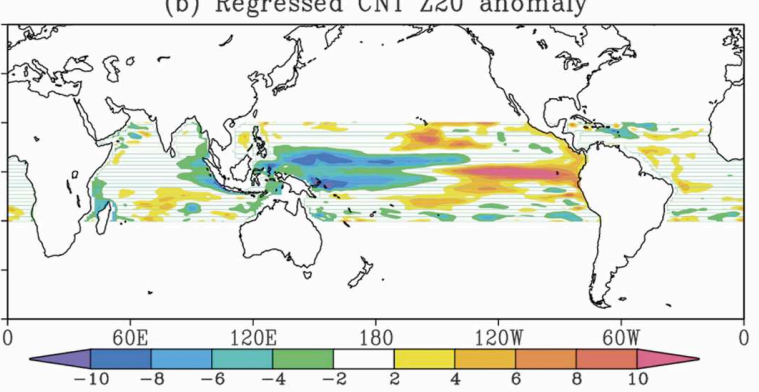

(c) Regressed CNT Uo anomaly

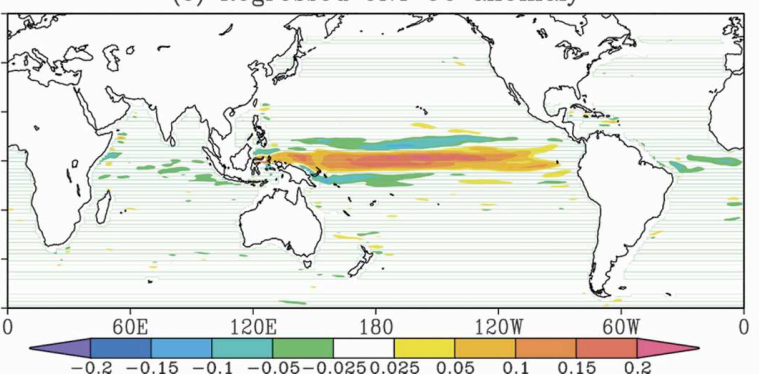

(d) Regressed BV SST

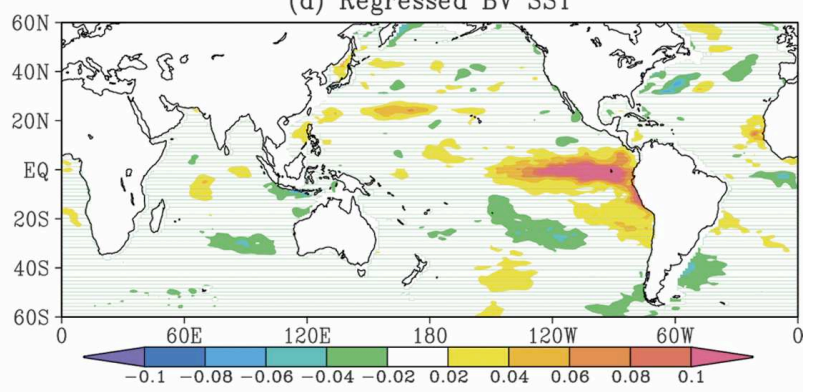

(e) Regressed BV Z20

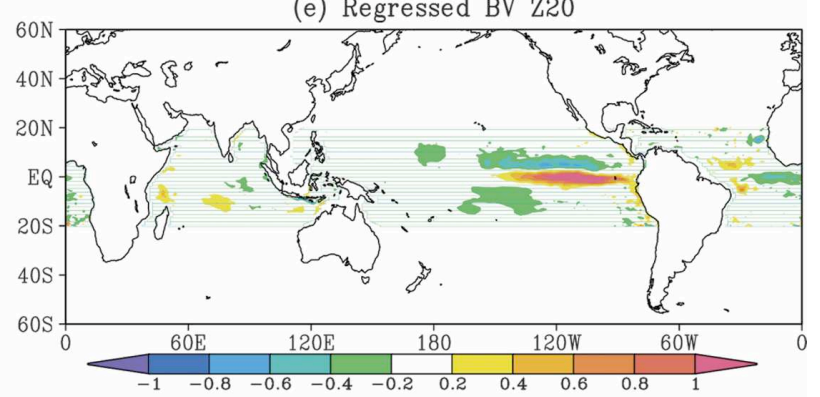

(f) Regressed BV Uo

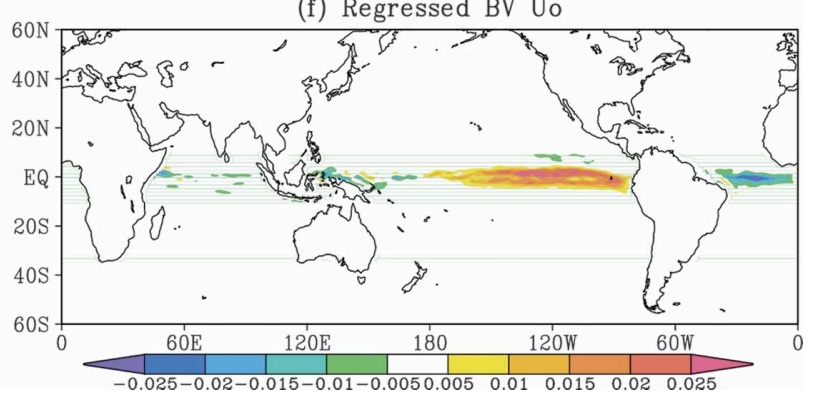

IG. 4. Oceanic regression maps in the global domain: (left) the background fields and (right) the BV fields. (a) SST anomaly $\left({ }^{\circ} \mathrm{C}\right)$; $Z_{20}$ anomaly (m); (c) surface zonal current anomaly $\left(\mathrm{m} \mathrm{s}^{-1}\right)$; (d) BV SST $\left({ }^{\circ} \mathrm{C}\right)$; (e) BV $Z_{20}$ (m); and (f) BV surface zonal current $\mathrm{s}^{-1}$ ). Background fields are regressed with the background Niño-3 index and BV fields are regressed with the BV Niño-3 index. The es of BV fields are arbitrary, but the ratio among BV variables (both oceanic and atmospheric variables) is retained as in the jinal. The background $Z_{20}$ anomaly and $\mathrm{BV} Z_{20}$ are only plotted within $20^{\circ} \mathrm{N} / \mathrm{S}$, since $Z_{20}$ is defined as the depth of the $20^{\circ}$ isotherm, $\mathrm{t}$ is not well defined beyond the Tropics.

ns in the BV fields have some features in common $\mathrm{h}$ the patterns of the background state, such as the sterly wind perturbations located in the central equaial Pacific and the high-low pattern in the BV sure pressure field. The baroclinic structure in the ght fields corresponds to the location of BV SST in . $4 \mathrm{~d}$. In addition, the BV outgoing radiation reflects enhanced convection activity in the eastern basin. is atmospheric structure, implying that an amplified turbation in the eastern Pacific induces a westerly al wind perturbation, indicates unstable air-sea inaction in the eastern Pacific. These features suggest it the bred perturbation is related to the coupled tability and therefore that we can refer it to as the upled BV mode. present in coupled GCMs, that is, the simulated SST variability in the eastern Pacific is smaller than the observed variability. Rienecker et al. (2000) suggest that such deficiencies may be associated with the atmospheric circulation in the tropical eastern Pacific not representing well the Walker circulation, which also limits the spread of ensemble SSTs. The high-low pressure patterns in Figs. 5f,g suggest that the coupled BV is able to reflect the impact of coupled instability upon the background atmospheric circulation by perturbing the longitudinal Walker circulation along the equator.

It is of interest to point out that the coupled $\mathrm{BV}$ also reflects the sensitivities in extratropical regions associated with background ENSO atmospheric teleconnections. Shown in Fig. 6 are the regression maps of surface 
(a) CNT Surface Pressure

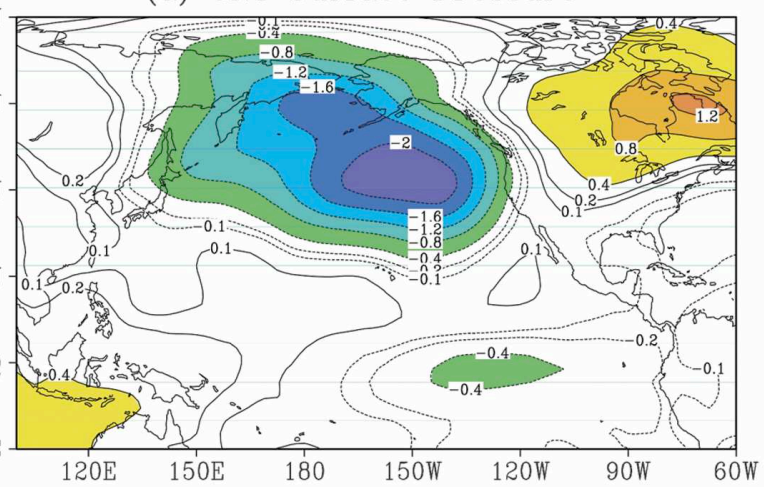

(b) CNT Geopotential at $200 \mathrm{mb}$

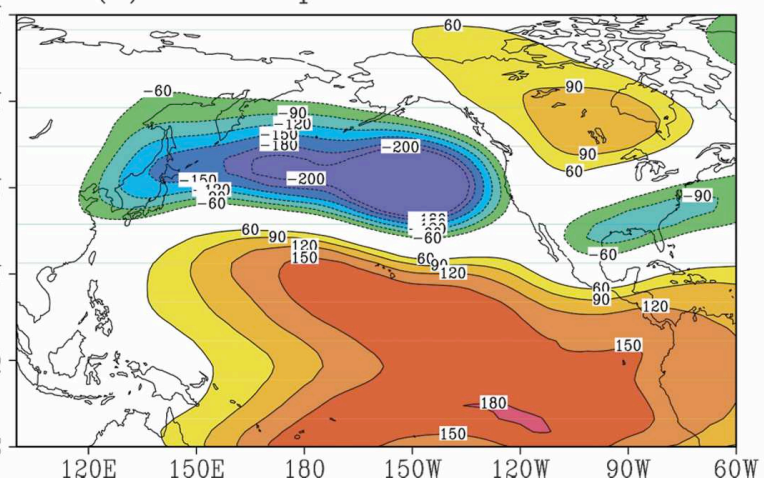

(c) BV Surface Pressure

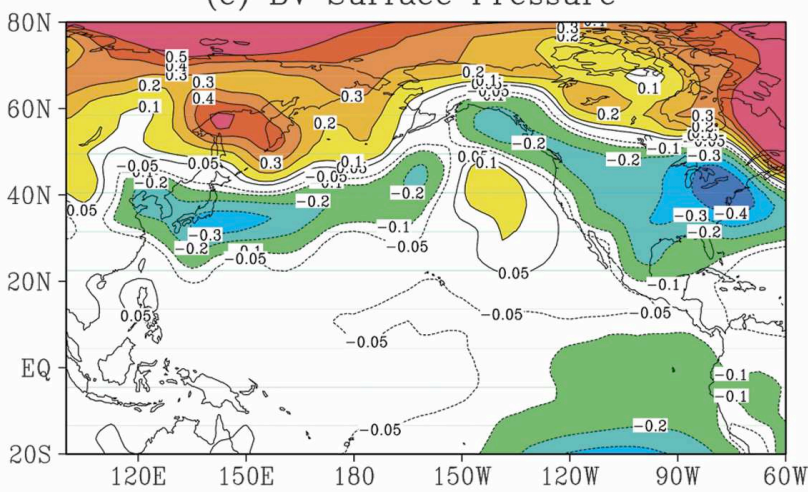

(d) BV Geopotential at $200 \mathrm{mb}$

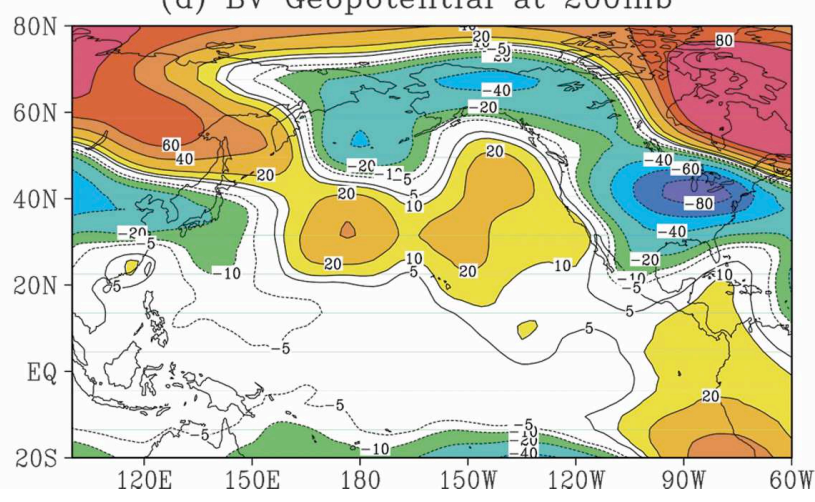

IG. 6. As in Fig. 3 except for atmospheric regression maps over the Pacific portion of the Northern Hemisphere: (a) background face pressure anomaly $(\mathrm{hPa})$; (b) background geopotential anomaly at $200 \mathrm{hPa}\left(\mathrm{m}^{2} \mathrm{~s}^{-2}\right)$; (c) BV surface pressure (hPa); and (d) BV potential at $200 \mathrm{hPa}\left(\mathrm{m}^{2} \mathrm{~s}^{-2}\right)$.

potential use for ensemble forecasting, particularly the purpose of effectively perturbing the coupled ISO processes in the tropical Pacific.

\section{Comparison of the NSIPP and the NCEP bred vectors}

Similar breeding experiments were carried out with S03 coupled model. The atmospheric component s the current version of medium-range forecast RF) global model with a spectral truncation of 62 ves (T62) in the horizontal (equivalent to nearly 200 ) and 64 vertical levels in sigma coordinate (Kanatsu et al. 1991; Saha et al. 2004). The ocean compoat is the Geophysical Fluid Dynamics Laboratory FDL) Modular Ocean Model V.3 (MOM3) with 40 ers in the vertical (Pacanowski and Griffies 1998). e zonal resolution is $1^{\circ}$ and the meridional resolution $1 / 3^{\circ}$ between $10^{\circ} \mathrm{S}$ and $10^{\circ} \mathrm{N}$, gradually increasing ough the Tropics until it is fixed at $1^{\circ}$ poleward of $\mathrm{S}$ and $30^{\circ} \mathrm{N}$.

Two independent breeding experiments were permed by choosing the last $4 \mathrm{yr}$ from a $23-\mathrm{yr}$ perfect period includes a warm event, which matures at model year 21 , two years after starting the breeding run. The rescaling factor for NCEP bred perturbations is based on the SST norm computed over the whole tropical belt $\left(10^{\circ} \mathrm{S}-10^{\circ} \mathrm{N}\right)$, not just in the tropical Pacific Niño-3 region like in the NSIPP BV. The perturbation size for the NCEP system was similar $\left(0.1^{\circ} \mathrm{C}\right)$ and the rescaling period of one month was the same as in the breeding experiments performed with the NSIPP CGCM. Like the NSIPP coupled experiments, the two BV runs for the NCEP system were very similar despite having been started with different random perturbations so that their results are processed as a single 8-yr time series. Comparisons between the results from the NSIPP and the NCEP coupled system are now presented showing the extent to which bred vectors are sensitive to the coupled GCMs. ${ }^{1}$

We first compare the ENSO characteristics in the background runs of the two coupled GCMs. Figures

\footnotetext{
${ }^{1}$ Unfortunately, the experiments performed at NCEP were erased, so we have only a limited number of diagnostic compari-
} 
BV SST EOF $(1-10)$ vs. |CNT Nino3|

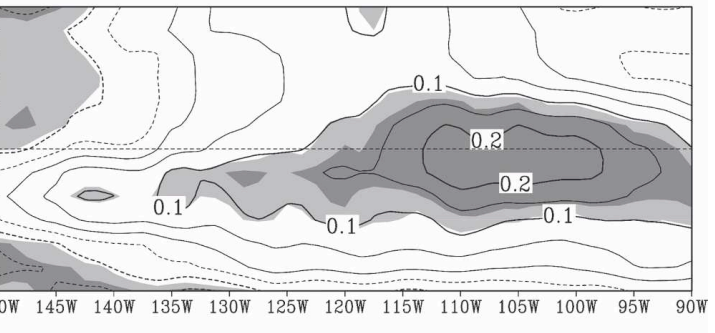

BV Hs EOF $(1-10)$ vs. |CNT Nino3|

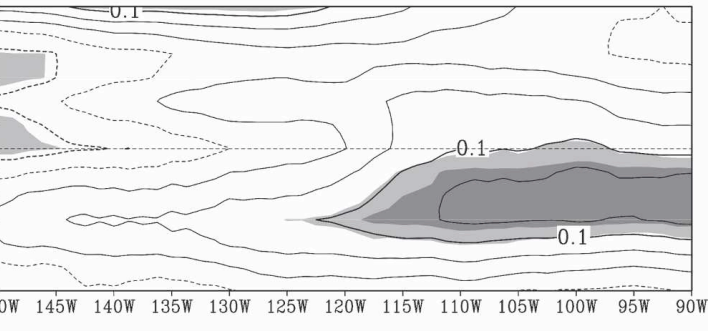

BV Taux EOF(1-10) vs. |CNT Nino3|

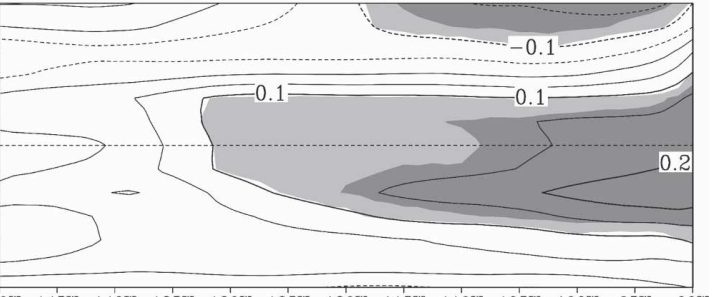

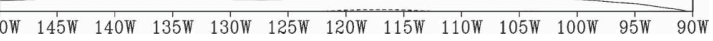

7. Lead/lag correlation maps along the equator for BV fields, reconstructed with the first 10 EOF modes, against olute value of the background Niño-3 index: (a) SST $\left({ }^{\circ} \mathrm{C}\right)$; face height $(\mathrm{m})$; and $(\mathrm{c})$ zonal wind stress $\left(\mathrm{N} \mathrm{m}^{-2}\right)$. The dark) gray shading denotes the significance level higher $\%(95 \%)$.

re the background oceanic regression maps showlat the oceanic components from both CGCMs ssfully reproduce the fundamental features of . However, they also have differences reflecting ences of numerical schemes in the model dynamdifferent choices of physical parameterizations. neridional structure of warming and thickening e height (Figs. 8d,e) in the NCEP/CFS03 GCM in astern Pacific is wider than that of the NSIPP (Figs. 8a,b). In addition, the regressed surface t of NCEP/CFS03 shows the southern branch of oaling patterns off the equator extending farther ward instead of being meridionally limited as in SIPP case. This can also be seen in the SST and current patterns.

spite these differences in the background, the rectors from the two coupled systems have major ground ENSO. To compare BV structures, we show the leading EOFs of oceanic variables. Figures $9 \mathrm{a}-\mathrm{c}$ are the first EOF mode of the BV SST and the first two modes for the BV thermocline from the NSIPP CGCM; Figs. 9d-f are the same modes using the BV from NCEP. Despite the fact that these are two different CGCMs with significantly different background evolution, there is a strong resemblance between the $\mathrm{BV}$ EOF modes. Both of the leading modes (EOF1) in NSIPP and NCEP bred vectors based on SST show an ENSOassociated warm feature in the tropical eastern Pacific farther east than in their respective background. Recalling that bred perturbations are rescaled in different regions (Niño-3 region for NSIPP and the complete tropical belt including three ocean basins for NCEP), the similarity observed in Figs. 9a and 9d indicates that the tropical Pacific dominates the growth of the BVs. However, reflecting the different mean structures and background ENSO variabilities from the two coupled systems, the NCEP BV EOF1 extends farther in space, covering the whole Niño-3 domain, while the NSIPP BV EOF1 is confined to east of $130^{\circ} \mathrm{W}$ and is limited in the meridional direction. The EOF1 modes representing ENSO variability explain $11 \%$ and $14 \%$ of the total variance of the growing SST perturbations in the NSIPP and NCEP models, respectively. Recall that the coupled growing perturbations include those due to a wide range of instabilities that appear in a coupled model. The fact that the leading EOF modes from BV fields in both coupled systems show a similar ENSOlike structure confirms our conjecture that the breeding method is capable of capturing the coupled instability even in the presence of other types of instabilities in the fully coupled GCM model. Moreover, this mode is robust and dominant since it is reproducible with different CGCMs and points out that the equatorial eastern Pacific is the most dynamically sensitive region for the growth of SST perturbations. Such a result indicates that the breeding method can help to identify the growing coupled instability related to the ENSO variability in a global coupled model.

Similar natural sensitivities in the eastern Pacific can also be found in the $\mathrm{BV}$ thermocline fields of both systems (Figs. 9b,c,e,f). For the background thermocline evolution (not shown), there are two dominant EOF modes related to the ENSO evolution: the leading one has a large variance in the eastern Pacific and the second one, representing an earlier transition, shows the anomalous deepening that starts from the subsurface of the western Pacific. We also examine the first two EOF modes for the BV thermocline. The result shows that 
(a) NSIPP regressed BV geopotential height at 500hpa

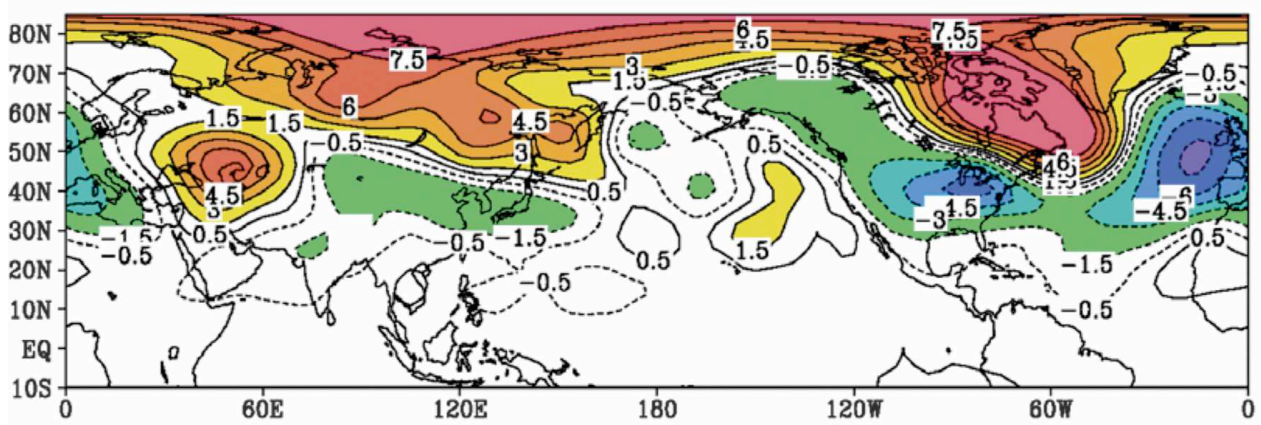

(b) CFS03 regressed BV geopotential height at 500hpa

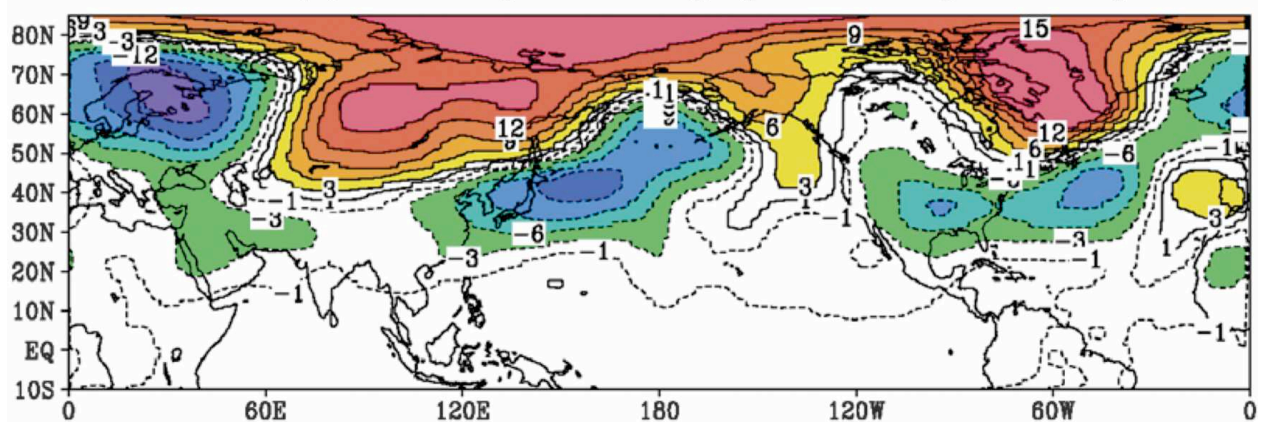

FIG. 10. Atmospheric regression maps in the Northern Hemisphere of BV sea level pressure (hPa)

(a) for NSIPP and (b) for NCEP/CFS03. Both fields are computed against their own BV Niño-3 indices.

the NSIPP coupled model under a perfect model nario.

The results show that the breeding method can ob$n$ the growing coupled modes associated with ENSO iability and that the BV growth rate is sensitive to background ENSO evolution, with the maximum ding the warm/cold events by three months. This d time in our results (compared with 6-12 months the ZC model) may seem to be too short for imving the current ensemble forecast system. Howr, we believe that this relatively short lead time is tially due to the fact that the ENSO cycle in this long ulation is more biennial than 3-7 years. The biennial ie scale shortens the "growing season" of bred vec$s$ because of the relatively fast pace of the evolving kground state. We would expect to obtain a longer d time from a BV growth rate derived from the IPP operational system since it will reflect the real eanic memory from the observations during initialtion, although the shorter time lag may be also asiated with the presence of other weather and ocec instabilities. We have explored this question with current NSIPP operational system, which includes 1 ocean observations, and where we used as back- indicate that the lead time is lengthened by about 2 months.

One of the main tasks in our study has been to extract the physical growing mode from the total growth variability, separating the ENSO signal from noise associated with weather and other less relevant instabilities. We used regression BV maps to illustrate that the ENSO-associated features can be captured by the breeding method. For the oceanic fields, the variability mainly comes from the tropical eastern Pacific, where the background anomalies have large variance due to the mean structure of the thermocline. We also show that the atmospheric BV in the Tropics carries the coupled features reflecting the growing perturbations at the boundary due to the unstable air-sea interaction. Such structure exhibits a longitudinal Walker-like circulation along the equator. Evidence from the lead/lag correlation maps suggests that such growing perturbation is related to the background ENSO variability. In addition to the coupled characteristics shown in the tropical domain, the extratropical circulation anomalies associated with the coupled BV display a wave-train teleconnection pattern over the North Pacific and North America, areas known to be strongly telecon- 
robustness of the coupled $\mathrm{BV}$ modes has been nstrated by comparing bred vectors derived from SIPP CGCM and NCEP/CFS03 CGCMs. The two ed GCMs can both represent the main features of NSO events, but differences exist in the detailed ure. Our results indicate that the coupled BV ure can be reproduced by the different CGCMs, ting very similar structure in their leading EOF s. In addition, the differences between the leading modes obtained from the two systems reflect that nherit the characteristics of the CGCMs. Strong blance between these two independent experican be found in many fields, even in those atmoic teleconnected regions associated with backd ENSO development. Our results suggest that lecting physically meaningful breeding paramone can use the breeding method to isolate the y varying, coupled instabilities from the fastng weather signals in a coupled GCM. The global ivities associated with the coupled instability inifrom the tropical Pacific can be retained even $\mathrm{h}$ the rescaling is simply done in the tropical $\mathrm{Pa}$ -

ng the same NSIPP CGCM, Kleeman et al. (2003) ated a climate-relevant leading singular vector by a tangent linear propagator derived from the subspanned by five dominant correlation EOF s of the background SST anomaly. In their work, ocused on the SST perturbation, so we can only are the SST structure from the two methods. initial singular vector shows a large warming perion in the tropical Pacific with a maximum loin the central Pacific. Their final singular vector ix months is very similar to our ocean regression (Fig. 3d) with a strong amplitude in the eastern c representing the mature state of ENSO. We do ave a warming feature in the northwest of the $c$ off the equator that appears in the initial singuctor. In addition, there is no clear evidence showlat the singular vector is sensitive to the ENSO $\mathrm{s}$ even though the growth rates from both methlow dependence on ENSO evolution. The simibetween the bred vector and the final singular (but not the initial singular vector) is also charstic of the results obtained with the ZC model t al. 2003; Xue et al. 1997b).

results indicate that the coupled $\mathrm{BVs}$ could be in ensemble forecasting as initial perturbations ffectively project on the ENSO-related large-scale es. Therefore, the next stage of our research is to y these methods in the NSIPP operational sysIn particular, we plan to test whether ensemble more effective than current ensembles using uncoupled perturbations introduced into the atmosphere or the ocean model components.

Acknowledgments. We greatly appreciate many valuable discussions with Max. J. Suarez. We are very grateful to Sonya Kulkarni Miller for much technical support. We are grateful for the constructive comments and suggestions made by three anonymous reviewers that considerably improved the manuscript. The NSIPP component of this work has been supported by grants from the NASA Seasonal-to-Interannual Prediction Project (NASA-NAG-55825 and NASA-NAG-12418).

\section{REFERENCES}

Bacmeister, J., and M. J. Suarez, 2002: Wind stress simulations and the equatorial momentum budget in an AGCM. $J$. Atmos. Sci., 59, 3051-3073.

_ S. D. Schubert, M. Suarez, and P. J. Pegion, 2000: Atlas of seasonal means simulated by the NSIPP 1 atmospheric GCM. Technical Report Series on Global Modeling and Data Assimilation 104606, 17, 194 pp.

Battisti, D. S., 1988: The dynamics and thermodynamics of a warm event in a coupled tropical atmosphere-ocean model. J. Atmos. Sci., 45, 2889-2919.

Boffetta, G., A. Crisanti, F. Papatella, A. Provenzale, and A. Vulpiani, 1998: Slow and fast dynamics in coupled systems: A time series analysis view. Physica D, 116, 301-312.

Cai, M., 1995: A simple model for the climatology and ENSO of equatorial Pacific ocean-atmosphere system. Proc. 20th Annual Climate Diagnostics Workshop, Seattle, WA, NOAA/ CPC, 314-317.

- 2003: Formation of the cold tongue and ENSO in the equatorial Pacific Basin. J. Climate, 16, 144-155.

— E. Kalnay, and Z. Toth, 2003: Bred vectors of the ZebiakCane model and their application to ENSO predictions. $J$. Climate, 16, 40-55.

Cane, M. A., S. E. Zebiak, and S. C. Dolan, 1986: Experimental forecasts of El Niño. Nature, 321, 827-832.

_ oscillations of the tropical ocean-atmosphere system. Part I: Linear analysis. J. Atmos. Sci., 47, 1562-1577.

Chen, D., M. A. Cane, A. Kaplan, S. E. Zebiak, and D. Huang, 2004: Predictability of El Niño over the past 148 years. $\mathrm{Na}$ ture, 428, 733-736.

Chen, Y.-Q., D. S. Battisti, T. N. Palmer, J. Barsugli, and E. S. Sarachik, 1997: A study of the predictability of tropical Pacific SST in a coupled atmosphere-ocean model using singular vector analysis: The role of annual cycle and the ENSO cycle. Mon. Wea. Rev., 125, 831-845.

Contreras, R. F., 2002: Long-term observations of tropical instability waves. J. Phys. Oceanogr., 32, 2715-2722.

Dijkstra, H. A., and J. D. Neelin, 1999: Coupled process and the tropical climatology. Part III: Instabilities of the fully coupled climatology. J. Climate, 12, 1630-1643.

Evans, E., N. Bhatti, J. Kinney, L. Pann, M. Peña, S.-C. Yang, E. Kalnay, and J. Hansen, 2004: RISE: Undergraduates find that regime changes in Lorenz's model are predictable. Bull. Amer. Meteor. Soc., 85, 520-524. 
2000: How predictability depends on the nature of uncertainty in initial conditions in a coupled model of ENSO. $J$. Climate, 13, 3298-3313.

F.-F., 1996: Tropical ocean-atmosphere interaction, the Pacific cold tongue, and the El Niño/Southern Oscillation. Science, 274, 76-78.

-, 1997: An equatorial ocean recharge paradigm for ENSO. Part I: Conceptual model. J. Atmos. Sci., 54, 811-829.

namitsu, M., and Coauthors, 1991: Recent changes implemented into the global forecast system at NMC. Wea. Forecasting, 6, 425-435.

eman, R., Y. Tang, and A. M. Moore, 2003: The calculation of climatically relevant singular vectors in the presence of weather noise as applied to the ENSO problem. J. Atmos. Sci., 60, 2856-2868.

ter, R. D., and M. J. Suarez, 1992: Modeling the land-surface boundary in climate models as a composite of independent vegetation stands. J. Geophys. Res., 97, 2697-2715.

if, M., and Coauthors, 1998: A review of predictability and prediction of ENSO. J. Geophys. Res., 103, 14 375-14 393.

ore, A. M., and R. Kleeman, 1996: The dynamics of error growth and predictability in a coupled model of ENSO. Quart. J. Roy. Meteor. Soc., 122, 1405-1446.

-, and —, 1997: The singular vectors of a coupled oceanatmosphere model of ENSO. Part II: Sensitivity studies and dynamical significance. Quart. J. Roy. Meteor. Soc., 123, 9831006.

-, and —_, 1999a: Stochastic forcing of ENSO by the intraseasonal oscillation. J. Climate, 12, 1199-1220.

-, and _ 1999b: The non-normal nature of El Niño and intraseasonal variability. J. Climate, 12, 2965-2982.

-, and —, 2001: The differences between the optimal perturbations of coupled models of ENSO. J. Climate, 14, 138163.

anowski, R. C., and S. M. Griffies, 1998: MOM 3.0 manual. NOAA/Geophysical Fluid Dynamics Laboratory, Princeton, NJ.

a, M., and E. Kalnay, 2004: Separating fast and slow modes in coupled chaotic systems. Nonlinear Processes Geophys., 11, 319-327.

land, C., and P. D. Sardeshmukh, 1995: The optimal growth of tropical sea surface temperature anomalies. J. Climate, 8, 1999-2024.

necker, M., M. Suarez, D. Admec, R. Koster, S. Schubert, and J. Hansen, 2000: NASA Seasonal-to-Interannual Project (NSIPP) annual report for 2000. [Available online at http:// nsipp.gsfc.nasa.gov/pubs/pubs_main.html.]

a, S., W. Wang, S. Nadiga, H.-L. Pan, and G. White, 2004: The new seasonal forecast model at NCEP. Preprints, 13th Conf. on Interactions of the Sea and Atmosphere, Portland, ME, Amer. Meteor. Soc., CD-ROM, 3.8.

Schopf, P. S., and M. J. Suarez, 1988: Vacillations in a coupled ocean-atmosphere model. J. Atmos. Sci., 45, 549-566.

_ , and A. Loughe, 1995: A reduced-gravity isopycnal ocean model: Hindcasts of El Niño. Mon. Wea. Rev., 123, 28392863.

Stockdale, T. N., D. L. T. Anderson, J. O. S. Alves, and M. A. Balmaseda, 1998: Global seasonal rainfall forecasts using a coupled ocean-atmosphere model. Nature, 392, 370-373.

Suarez, M. J., and P. S. Schopf, 1988: A delayed action oscillator for ENSO. J. Atmos. Sci., 45, 3283-3287.

Thompson, C. J., 1998: Initial conditions for optimal growth in a coupled ocean-atmosphere model of ENSO. J. Atmos. Sci., $\mathbf{5 5}, 537-557$.

Toth, Z., and E. Kalnay, 1993: Ensemble forecasting at NMC: The generation of perturbations. Bull. Amer. Meteor. Soc., 74, 2317-2330.

— them? Idojaras, 100, 43-52.

— ing method. Mon. Wea. Rev., 125, 3297-3319.

Van der Vaart, P. C. F., H. A. Dijkstra, and F.-F. Jin, 2000: The Pacific cold tongue and the ENSO mode: A unified theory within the Zebiak-Cane model. J. Atmos. Sci., 57, 967-988.

Vialard, J., F. Vitart, M. A. Balmaseda, T. N. Stockdale, and D. L. Anderson, 2003: An ensemble generation method for seasonal forecasting with an ocean-atmosphere coupled model. ECMWF Tech. Memo. 417, 20 pp.

Vintzileos, A., M. M. Rienecker, M. J. Suarez, S. K. Miller, P. J. Pegion, and J. T. Bacmeister, 2003: Simulation of the El Niño-Interannual Prediction Project coupled general circulation model. CLIVER Exchanges, Vol. 8, No. 4, International CLIVAR Project Office, Southampton, United Kingdom, $25-27$.

Xue, Y., M. A. Cane, S. E. Zebiak, and M. B. Blumenthal, 1994: On the prediction of ENSO: A study with a low order Markov model. Tellus, 46A, 512-528.

,-- , and -1997 a: Predictability of a coupled model of ENSO using singular vector analysis. Part I: Optimal growth in seasonal background and ENSO cycles. Mon. Wea. Rev., 125, 2043-2056.

,-- , and $-1997 \mathrm{~b}$ : Predictability of a coupled model of ENSO using singular vector analysis. Part II: Optimal growth and forecast skill. Mon. Wea. Rev., 125, 2057-2073.

Yang, S., K. Lau, and P. Schopf, 1999: Sensitivity of the tropical Pacific Ocean to precipitation induced freshwater flux. Climate Dyn., 15, 737-750.

Zebiak, S. E., and M. A. Cane, 1987: A model El Niño-Southern Oscillation. Mon. Wea. Rev., 115, 2262-2278. 